Rev Inv Vet Perú 2019; 30(2): 598-604

http://dx.doi.org/10.15381/rivep.v30i2.16073

\title{
Niveles de leptina sérica en aves comerciales
}

\author{
Serum leptin levels in poultry
}

José Henry Osorio ${ }^{1,2,4}$, Jorge Abel Castañeda ${ }^{3}$

\section{Resumen}

El objetivo del estudio fue determinar los niveles de leptina en pollos y gallinas ponedoras mediante el uso de un kit para uso de muestras de humano. Se colectaron muestras de sangre en ayunas de 80 pollos de engorde de la línea Cobb 500 de 4 semanas (40 hembras y 40 machos) y de 86 gallinas de 26 semanas (43 Lohmann Brown y 43 Hyline). Se determinaron los niveles de leptina mediante inmunoensayo enzimático tipo sandwich, previa validación del kit para su uso en aves comerciales. Los valores promedio, mínimo, máximo y desviación estándar de leptina para pollos de engorde fueron de $0.61,0.12,3.99$ y $0.10 \mathrm{ng} / \mathrm{ml}$, respectivamente, sin diferencia significativa entre sexos. Las gallinas ponedoras reportaron valores promedio mínimo, máximo y desviación estándar de $0.66,0.16,1.83$ y $0.11 \mathrm{ng} / \mathrm{ml}$, respectivamente, sin diferencia significativa entre líneas. En forma similar, tampoco se encontró diferencia estadística entre pollos de engorde y gallinas ponedoras. Los resultados del estudio indican que los valores de referencia para leptina en pollos y gallinas son de $0.63 \mathrm{ng} / \mathrm{ml}$; asimismo, que pueden ser medidos en aves comerciales mediante el uso del kit humano.

Palabras clave: adipocitoquinas; aves; inmunoensayo; leptina; metabolismo

\section{Abstract}

The aim of this study was to determine the levels of leptin in broilers and laying hens by using a kit designed for human samples. Fasting blood samples were collected from 80 broilers of the Cobb 500 line of 4 weeks of age ( 40 females and 40 males) and 86 hens of 26 weeks of age (43 Lohmann Brown and 43 Hy-line). Leptin levels were determined by an ELISA sandwich, after validation of the kit for use in commercial poultry. The mean,

\footnotetext{
${ }^{1}$ Laboratorio de Investigación en Bioquímica Clínica y Patología Molecular, Departamento de Ciencias Básicas de la Salud, Universidad de Caldas, Manizales, Colombia

${ }^{2}$ Laboratorio de Investigación en Metabolismo, Universidad de Manizales, Colombia

${ }^{3}$ Facultad de Ciencias Exactas y Naturales, Universidad de Caldas, Manizales, Colombia

${ }^{4}$ E-mail: jose.osorio_o@ucaldas.edu.co
}

Recibido: 31 de julio de 2018

Aceptado para publicación: 28 de marzo de 2019 
minimum, maximum and standard deviation values of leptin for broilers were $0.61,0.12$, 3.99 and $0.10 \mathrm{ng} / \mathrm{ml}$, respectively, without significant difference between sexes. Laying hens reported a mean, minimum, maximum and standard deviation values of $0.66,0.16$, 1.83 and $0.11 \mathrm{ng} / \mathrm{ml}$, respectively, without significant difference between lines. Similarly, no statistical difference was found between broiler chickens and laying hens. The results of the study indicate that the reference values for leptin in chickens and chickens are 0.63 $\mathrm{ng} / \mathrm{ml}$, and the levels can be measured by using the human kit.

Key words: adipocytokines; birds; immunoassay; leptin; metabolism

\section{INTRODUCCIÓN}

La leptina es una hormona producida en el tejido adiposo de las aves (Sánchez-Muñoz et al., 2005; Radin et al., 2009; Gómez et al., 2011), pero también en otros órganos como placenta, ovarios, músculo esquelético, estómago, hígado y glándula pituitaria (Muoio y Lynis, 2002; Ishioka et al., 2006). Su concentración sérica está relacionada directamente con la cantidad de grasa corporal (Considine et al., 1996; Ishioka et al., 2007; Mazaki-Tovi et al., 2010); además, está relacionada con la actividad de la lipoproteinlipasa (LPL) (Flynn y Plata-Salaman, 1999). A nivel del cerebro y en tejidos actúa uniéndose a sus receptores específicos (Ob-RS) (Bjorbaek et al., 1998). La leptina regula tanto la saciedad a través de los receptores hipotalámicos específicos como el balance energético, la eficiencia energética y, además, contribuye a la adaptación a la inanición (Taouis et al., 1998). Así mismo, tiene efectos metabólicos al disminuir la secreción del neuropéptido Y (NPY) (Tartaglia et al., 1995).

Esta adipocitoquina induce un efecto sensibilizador a la insulina, al promover la oxidación de ácidos grasos libres y la reducción de la acumulación de grasa ectópica en tejidos no adiposos. Se han encontrado receptores de leptina en células $\beta$ pancreáticas, donde la leptina puede incidir en la regulación y producción de insulina (Benomar et $a l ., 2000)$. La infusión de leptina recombi- nante en un páncreas aislado inhibe considerablemente la secreción de la insulina producida por la vía de la acetilcolina (Schwartz et al., 1996). Varios trabajos indican que esta adipocitoquina tiene funciones similares en el pollo como en los mamíferos (Li et al., 2011). Se ha demostrado que la inyección intracerebroventricular de leptina en pollos de engorde y gallinas ponedoras disminuye el consumo de alimento (Denbow et al., 2000).

En el presente estudio se validó el uso del kit humano de leptina para la medición de leptina en aves comerciales y se buscó la posible diferencia de los valores de leptina sérica entre sexos en pollos de engorde, así como la posible diferencia entre pollo de engorde y gallinas ponedoras.

\section{Materiales y Métodos}

\section{Animales}

Se obtuvo muestras de sangre en ayunas, mediante punción en la vena yugular, de 80 pollos de engorde de la línea Cobb 500 de 4 semanas (40 hembras y 40 machos) y de 86 gallinas de 26 semanas de edad (43 Lohmann Brown y 43 Hy-Line). Las muestras se transportaron en cadena de frío al laboratorio para la obtención del suero mediante centrifugación a $2300 \mathrm{~g}$ por 5 minutos. Las aves fueron criadas en ambiente controlado en la vereda Morrogordo, finca El Remanso, ubicada en el Municipio de Manizales, Co- 
lombia, a $1800 \mathrm{msnm}$ y con una temperatura promedio de $23^{\circ} \mathrm{C}$. El estudio fue aprobado por el Comité de Ética de la Universidad de Manizales, según acta 001 de 2017.

\section{Análisis de Leptina}

Las muestras fueron analizadas inmediatamente a la obtención de los sueros. Para la determinación de la leptina se utilizó la prueba de ELISA tipo sandwich en fase sólida (Invitrogen ${ }^{\mathrm{TM}}$ Leptin Human ELISA kit), donde un anticuerpo monoclonal específico para la leptina humana ha sido revestido sobre los pocillos de las tiras de microtitulación proporcionadas. Se siguió el procedimiento indicado por el laboratorio fabricante. La intensidad de color producido en los pocillos es directamente proporcional a la concentración de leptina presente en la muestra original. La lectura se hizo en un equipo lector de micro ELISA (TitertekMultiscan ${ }^{\mathrm{TM}}$ ) a una absorbancia de $450 \mathrm{~nm}$. Las absorbancias obtenidas de los estándares se grafican junto con las concentraciones, y de la curva de calibración se obtienen las concentraciones de leptina de las respectivas muestras.

\section{Análisis Estadístico}

Los resultados fueron analizados a través del programa estadístico IBM SPSS Statistics 23, considerando diferencia estadística significativa con valores $\mathrm{p}<0.05$.

\section{Validación del Método}

Para la validación del uso del kit humano en aves comerciales se tuvo en cuenta la precisión, la exactitud y el límite de detección (Jensen, 2006; USDA et al., 2018).

- Precisión: El coeficiente de variación (CV) intra-ensayo fue calculado después de analizar 10 muestras ( 5 de pollos y 5 de gallinas) por cinco veces en un solo ensayo, obteniéndose un valor del $15 \%$. El coeficiente de variación (CV) interensayo fue determinado analizando las mismas muestras en cinco ensayos se- parados, obteniéndose un valor del $18 \%$.

- Exactitud: Se evaluó indirectamente por linearidad luego de preparar varias diluciones. Cinco muestras de gallinas y cinco muestras de pollos fueron diluidas con el correspondiente buffer de dilución utilizado en cada ensayo, obteniendo concentraciones de $0,20,50,80$ y $100 \%$, obteniendo los siguientes resultados: pollos: $\left(\mathrm{Y}=0.9437 \mathrm{X}+0.0342, \mathrm{r}^{2}=0.9997\right)$ y gallinas: $\left(\mathrm{Y}=0.9554 \mathrm{X}+0.0252, \mathrm{r}^{2}=\right.$ 0.9997).

- Límite de detección: Fue de $0.098 \mathrm{ng} / \mathrm{ml}$ para las muestras de pollos y de 0.095 $\mathrm{ng} / \mathrm{ml}$ para las muestras de las gallinas.

Durante los ensayos se trabajó además con 10 muestras de suero humano (punción en la vena radial y la muestra centrifugada a $2300 \mathrm{~g}$ por 5 minutos), obteniendo valores promedio, mínimo, máximo y desviación estándar de $2.17,1.85,3.84$ y $0.76 \mathrm{ng} / \mathrm{ml}$, respectivamente, con un CV de 10\% intra-ensayo y de $11 \%$ inter-ensayo.

\section{Resultados}

Los valores de leptina en pollos no presentaron diferencia estadística debido al sexo ( $p=0.316$, Cuadro 1). En forma similar, los valores para las gallinas Lohmann Brown y Hy-line fueron estadísticamente similares $(\mathrm{p}=0.725$, Cuadro 1$)$. Así mismo, los valores de leptina para pollos y gallinas fueron estadísticamente similares ( $\mathrm{p}=0.472$; Cuadro 1$)$.

\section{Discusión}

Los reactivos que se usaron para la medición de la leptina en las aves fueron de tipo humano debido a la alta homología entre distintas especies. Se sabe que los genes de leptina humana poseen $84 \%$ de homología con el ratón y 83\% con la rata (Maymó, 2010). La estructura de cristalización revela un paquete de cuatro hélices alfa antiparalelas, 
Cuadro 1. Valores de leptina en pollos de engorde y gallinas de postura

\begin{tabular}{lcccccc}
\hline & \multicolumn{3}{c}{ Pollos } & \multicolumn{3}{c}{ Gallinas } \\
\hline & $\begin{array}{c}\text { Macho } \\
(\mathrm{n}=40)\end{array}$ & $\begin{array}{c}\text { Hembra } \\
(\mathrm{n}=40)\end{array}$ & $\begin{array}{c}\text { Total } \\
(\mathrm{n}=80)\end{array}$ & $\begin{array}{c}\text { Lohmann } \\
\text { Brown } \\
(\mathrm{n}=43)\end{array}$ & $\begin{array}{c}\text { Hy-line } \\
(\mathrm{n}=43)\end{array}$ & $\begin{array}{c}\text { Total } \\
(\mathrm{n}=86)\end{array}$ \\
\hline Promedio & $0.66^{\mathrm{a}}$ & $0.56^{\mathrm{a}}$ & $0.60^{\mathrm{a}}$ & $0.68^{\mathrm{a}}$ & $0.64^{\mathrm{a}}$ & $0.66^{\mathrm{a}}$ \\
Mínimo & 0.12 & 0.13 & 0.12 & 0.16 & 0.28 & 0.16 \\
Máximo & 3.99 & 1.00 & 3.99 & 1.66 & 1.83 & 1.83 \\
D.E. & 0.10 & 0.10 & 0.46 & 0.11 & 0.12 & 0.44 \\
\hline
\end{tabular}

Valores con superíndices similares dentro de sexos de pollos, dentro de líneas de gallinas y dentro de tipos de aves (pollos vs. gallinas) no son estadísticamente diferentes ( $p>0.05$ )

cuatro giros beta y un enlace disulfuro entre los residuos aminoacídicos 117 y 167 . Es una estructura altamente conservada entre los mamíferos (Zhang et al., 1994). La mayoría de los estudios hechos en aves discuten la similitud del gen de leptina entre mamíferos y aves. Así, Taouis et al. (1998) reportan que el tamaño de la secuencia del gen de leptina del pollo tomado del hígado tiene una similitud del 97,96 y $83 \%$ con el ratón, rata y humano, respectivamente. Esta alta similitud, en términos porcentuales, hace posible, como en el presente estudio, el uso de kits comerciales para humanos, con buenos niveles de detección de este metabolito en las muestras de los animales estudiados.

Se conoce que la secuencia del gen de leptina en aves es $94.6 \%$ similar a la secuencia del gen leptina en ratones (Ninov et al., 2008), pero tiene un residuo de cisteína adicional que pueda afectar su estructura conformacional (Taouis et al., 1998; Ashwel et al., 1999). Así mismo, en el pollo se detectó exclusivamente la expresión de la leptina en el hígado, característica atribuible al metabolismo lipídico en el hígado que es el órgano principal de la lipogénesis en las aves (Ninov et al., 2008). Por otro lado, se sabe que la insulina, la corticosterona y los estrógenos en el pollo regulan esta expresión en el hígado, especie que además, tiene un crecimiento muy rápido, lo que conlleva a un exceso de grasa corporal, generando problemas en el desarrollo muscular y mortalidad (Taouis et al., 2001).

Una característica de la secuencia de la leptina del pollo es que esta presenta solamente 145 aminoácidos en lugar de 146 aminoácidos que tiene la secuencia en los mamíferos (Taouis et al., 1998; Ashwel et al., 1999; Taouis et al., 2001). Con relación al gen de leptina aviar existen controversias; sin embargo, se ha demostrado que existe un receptor funcional de leptina aviar, y se ha confirmado su capacidad para unir leptinas de diferentes vertebrados (Gogga et al., 2013).

Los valores de leptina obtenidos de forma general, sin discriminar sexo, en el presente estudio son menores si se compara con los descritos por Dridi et al. (2000), quienes determinan la leptina mediante radioinmunoensayo con un kit multi-especies y un kit específico para pollos, encontrado valores de $1.478 \mathrm{ng} / \mathrm{ml}$ a las 15 semanas, $1.485 \mathrm{ng} / \mathrm{ml} \mathrm{a}$ las 18 semanas y $2.709 \mathrm{ng} / \mathrm{ml}$ a las 22 semanas, concluyendo que los niveles plasmáticos de leptina aumentan con la edad; asimismo, encontraron diferencias entre aves con y sin 
ayuno. Por otro lado, se ha demostrado que en gallinas a las cuales se la ha suprimido el alimento durante 24 horas presentan niveles bajos de leptina a nivel del tejido adiposo (Sato et al., 2000); de allí que es posible que los menores valores de leptina hallados en el presente estudio se deban al tiempo de ayuno previo a la toma de la muestra de sangre. $\mathrm{Al}$ comparar los resultados de los niveles de leptina de este trabajo con otros, se observa que en gallinas que se encuentran en estado de ayuno, los niveles de leptina son considerablemente bajos,

En el playero común (Calidris alpina) se reportan valores de $2.42 \mathrm{ng} / \mathrm{ml}$ de leptina (rango: 1.19-3.83 ng/ml) (Gogga et al., 2013) y en el pato petrel picofino (Pachyptila belcheri) se encontraron valores entre 1 y 3 $\mathrm{ng} / \mathrm{ml}$ (Quillfeldt et al., 2009), valores que son mayores a los reportados en el presente trabajo. En el humano (Ostlund et al., 1996) y en ratones (Frederich et al., 1995) se han detectado diferencias significativas por efecto del sexo, edad y tipo de dietas, aunque la causa no ha sido debidamente identificada, pudiendo deberse a la síntesis de leptina en tejidos no-adiposos, como la placenta y la glándula mamaria (Masuzaki et al., 1997).

\section{Conclusiones}

- Los valores de referencia para leptina en pollos y gallinas son de $0.63 \mathrm{ng} / \mathrm{ml}$.

- El kit de leptina para uso humano puede ser utilizado en muestras séricas de pollos y gallinas.

\section{Literatura Citada}

1. Ashwell CM, Czerwinski SM, Brocht DM, McMurtry JP. 1999. Hormonal regulation of leptin expression in broiler chickens. Am J Physiol 276: 226-232.

2. Benomar Y, Taouis M, Rideau N. 2000. La leptine inhibe la secretiond'insuline du poulet. Nutr Clin Metab 14: 125-146.
3. Bjorbaek C, Elmquist JK, Michl P, Ahima RS, van Bueren A, McCall AL, Flier JS. 1998. Expression of leptin receptor isoforms in rat brain microvessels. Endocrinology 139: 3485-3491. doi: 10.1210/endo.139.8.6154

4. Considine RV, Sinha MK, Heiman ML, Kriauciunas A, Stephens TW, Nyce MR, Ohannesian JP, et al. 1996. Serum immunoreactive leptin concentrations in normal weight and obese humans. New Engl J Med 334: 292-295. doi: 10.1056/NEJM199602013340503

5. Denbow DM, Meade S, Robertson A, McMurtry JP, Richards M, Ashwell C. 2000. Leptin induced decrease in food intake in chickens. Physiol Behav 69: 359-362. doi: 10.1016/S00319384(99)00258-9

6. Dridi S, Raver N, Gussakovsky EE, Derouet M, Picard M, Gertler A, Taouis M. 2000. Biological activities of recombinant chicken leptin $\mathrm{C} 4 \mathrm{~S}$ analog compared with unmodified leptins. Am J Physiol-Endoc M 279: 116-123. doi: 10.1152/ajpendo.2000.279.1.E116

7. Flynn MC, Plata-Salaman CR. 1999. Leptin (OB protein) and meal size. Nutrition 15: 508-509.

8. Frederich RC, Hamann A, Anderson S, Löllmann B, Lowell BB, Flier JS. 1995. Leptin levels reflect body lipid content in mice: evidence for dietinduced resistance to leptin action. Nat Med 1: 1311-1314. doi: 10.1038/nm12951311

9. Gogga P, Karbowska J, Kochan Z, Meissner W. 2013. Circulating leptin levels do not reflect the amount of body fat in the dunlin Calidris alpina during migration. Gen Comp Endocr 187: 7478. doi: 10.1016/j.ygcen.2013.04.001

10. Gómez J, Córdoba GM, Guarín-Torres CS, Guarín-Torres MP. 2011. Incidencia de hígado graso y pododermatitis plantar en pollos comerciales en la planta de sacrificio de Pollo Olympico SA. Rev Cienc Anim 4: 41-57. 
11. Ishioka K, Hosoya K, Kitagawa H, Shibata H, Honjoh T, Kimura K, Saito M. 2007. Plasma leptin concentration in dogs: effects of body condition score, age, gender and breeds. Res Vet Sci 82: 1115. doi: 10.1016/j.rvsc.2006.06.002

12. Ishioka K, Omachi A, Sagawa M, Shibata H, Honjoh T, Kimura K, Saito M. 2006. Canine adiponectin: cDNA structure, mRNA expression in adipose tissues and reduced plasma levels in obesity. Res Vet Sci 80: 127-132. doi: 10.1016/j.rvsc.2005.05.011

13. Jensen AL. 2006. Validation of diagnostic tests in haematology laboratories. In: Feldman BF, Zinkl JG, Jain NC (eds). Schalm's Veterinary hematology. $5^{\text {th }}$ ed. Ames, Iowa: Blackwell Publishing. p 20-28.

14. Li R, Hu Y, Ni Y, Xia D, Grossmann $\boldsymbol{R}$, Zhao R. 2011. Leptin stimulates hepatic activation of thyroid hormones and promotes early posthatch growth in the chicken. Comp Biochem Physiol A Mol Integr Physiol 160: 200-206. doi: 10.1016/j.cbpa.2011.06.001

15. Masuzaki H, Ogawa Y, Sagawa N, Hosoda K, Matsumoto T, Mise H, Nishimura H, et al. 1997. Nonadipose tissue production of leptin: leptin as a novel placenta-derived hormone in humans. Nat Med 3: 1029-1033.

16. Maymó JL. 2010. Regulación de la expresión de leptina y su acción en células placentarias. Tesis doctoral. Buenos Aires, Argentina: Univ. de Buenos Aires. $232 \mathrm{p}$.

17. Mazaki-Tovi M, Feuermann Y, Segev G, Klement E, Yas-Natan E, Farkas $A, K o l$, et al. 2010. Increased serum leptin and insulin concentrations in canine hypothyroidism. Vet J 183: 109-114. doi: 10.1016/j.tvj1.2008.08.017

18. Muoio DM, Lynis DG. 2002. Peripheral metabolic actions of leptin. Best Pract Res Cl En 16: 653-666. doi: 10.1053/ beem. 2002.0223

19. Ninov K, Corrêa M, Javiel H, Millor FR, Nones K, Lehmann LC. 2008. Investigation of leptin gene in broiler and layer chicken lines. Sci Agr 65: 214-219. doi: 10.1590/S0103-90162008000200016

20. Ostlund RE, Yang JW, Klein S, Gingerich R. 1996. Relation between plasma leptin concentration and body fat, gender, diet, age, and metabolic covariates. J Clin Endocr Metab 81: 3909-3913. doi: 10.1210/jcem.81.11.8923837

21. Quillfeldt P, Everaert N, Buyse J, Masello JF, Dridi S. 2009. Relationship between plasma leptin-like protein levels, begging and provisioning in nestling thinbilled prions Pachyptila belcheri. Gen Comp Endocr 161: 171-178. doi: 10.1016/ j.ygcen.2008.12.006

22. Radin MJ, Sharkey LC, Holycross BJ. 2009. Adipokines: a review of biological and analytical principles and an update in dogs, cats, and horses. Vet Clin Path 38: 136-156. doi: 10.1111/j.1939165X.2009.00133.X

23. Sánchez-Muñoz F, García-Macedo R. Alarcón-Aguilar F, Cruza M. 2005. Adipocinas, tejido adiposo y su relación con células del sistema inmune. Gac Med Mex 141: 505-512.

24. Sato K, Nishida M, Takahashi T, Akiba Y. 2000. Nutritional regulation of leptin expression in adipose tissues of broiler chickens. In: XXI World's Poultry Congress. Montreal.

25. Schwartz MW, Baskin DG, Bukowski TR, Kuijper JL, Foster D, Lasser G, Prunkard DE, et al. 1996. Specificity of leptin action on elevated blood glucose levels and hypothalamic neutopeptide $Y$ gene expression in ob/obmice. Diabetes 45: 531-535.

26. Taouis M, Chen JW, Daviaud C, Dupont J, Derouet M, Simon J. 1998. Cloning the chicken leptin gene. Gene 208: 239-242. doi: 10.1016/S03781119(97)00670-7

27. Taouis $M$, Dridia $S$, Cassya $S$, Benomara Y, Raverb $N$, Rideaua $N$. 2001. Chicken leptin properties and actions. Domest Anim Endocrin 21: 319327. doi: 10.1016/S0739-7240(01)00122-9 
28. Tartaglia LA, Dembski M, Weng X, Deng N, Culpepper J, Devos R, Richards GJ, et al. 1995. Identification and expression cloning of a leptin receptor, OB-R. Cell 83: 1263-1271. doi: 10.1016/0092-8674(95)90151-5

29. [USDA] Department of Health and Human Services, Food and Drug Administration, [CDER] Center for Drug Evaluation and Research, [CVM] Center for Veterinary Medicine. 2018. Bioanalytical method validation.
Guidance for industry. [Internet]. Available in: https://www.-fda.gov/ down loads / drugs / guidances / ucm070107.pdf

30. Zhang Y, Proenca $R$, Maffei $M$, Barone M, Leopold L, Friedman JM. 1994. Positional cloning of the mouse obese gene and its human homologue. Nature 372: 425-432. doi: 10.1038/ $372425 \mathrm{a} 0$ 